\title{
Theoretical approach describing the thermal regime of snow-covered sea ice
}

\author{
NOBUO ONO, \\ National Institute of Polar Research, Tokyo 173, Japan \\ MAXIM S. KRASS \\ Geological Institute, Russian Academy of Science, Moscow, Russia
}

\begin{abstract}
As the greater part of sea-ice area is covered with snow, the thermal regime of sea ice is characterized by the thermal behavior of snow-covered sea ice. In this paper the thermal regime of snow-covered sea ice is quantitatively investigated with a one-dimensional non-linear boundary model which contains: compaction of snow cover; internal absorption of solar radiation; evaporation-condensation within snow cover; equilibrium phase change of brine within sea ice; and vertical oceanic heat flux from seawater to ice. Penetration of air temperature oscillations into the snow-covered sea ice increases remarkably with increasing snow density. As internal melting within the snow-covered sea ice appears with increasing solar radiation, the rise in air temperature and increase of solar radiation in the springtime produce a corresponding change in the thermal state of sea ice, causing a drastic retreat of seaice cover. A case study for warm sea ice is presented describing the thermal state during the melting season.
\end{abstract}

\section{INTRODUCTION}

Because sea ice is considered to be an early indicator of global warming, the thermal regime of sea ice poses one of the most interesting problems in sea-ice research. As the greater part of the sea-ice area is covered with snow, the thermal regime of sea ice is characterized by the thermal behavior of snow-covered sea ice.

There are several models accounting for snow cover on sea ice which use heat and mass balance of sea-ice cover and growth and decay of sea-ice zone (e.g. Maykut, 1986).

In the present paper we investigate a theoretical approach to snow cover on sea ice. It is well known that for short-wave solar radiation snow and ice are semitransparent. The effects of the snow and ice response to solar radiation, namely subsurface temperature maxima and internal melting possible under increasing radiative flux (particularly at springtime), are qualitatively obtained. These phenomena may cause a visible change in temperature distribution in snow and ice. Moreover, internal melting zones may play the role of energy sinks and sources that must be accounted for in the theoretical study of heat and mass balance of snow-covered sea ice.

The notion of thermal regime includes temperature fields and melting zones. Occurrence of melting zones within the sea-ice layer and on the boundary between overlying snow cover and sea ice (on the surface of sea ice) may determine the following significant properties of the thermal regime: a pattern of temperature distribution and redistribution of fluxes with depth; total heat-mass balance of the "snow-cover and sea-ice" system; and variations of behavioral characteristics of sea ice including the rapid decrease of its stability and subsequent possible decay.

\section{BASIC EQUATIONS}

The temperature field in snow and ice media is described by differential equations of heat and mass transfer containing terms which correspond to internal melting, absorption of short-wave solar radiation, and evaporation -condensation. We shall regard a two-layered system consisting of snow cover and sea ice (Fig. 1). The thermal processes occurring in the snow and ice layers vary due to differences between the snow and sea-ice fabrics, such as compaction and internal evaporation-condensation affecting only the snow. Salt impurities enclosed as brine in the snow and ice layers play an important role in the thermal behavior of the snow-covered sea ice. The freezethaw phase change in ice-brine mixture maintains the equilibrium concentration of brine with changing temperature and is the source and sink of latent heat. Inasmuch as thickness of snow and sea ice is small relative to areal extent of ice floes, we address the one-dimensional case with a single space variable $(z)$ directed downward.

The two optical parameters used here are albedo $(\alpha)$ and the attenuation coefficient of solar radiation $(b)$. The internal heat source function due to absorption of solar radiation penetrating the snow and ice is given by (Krass and Merzlikin, 1990) 


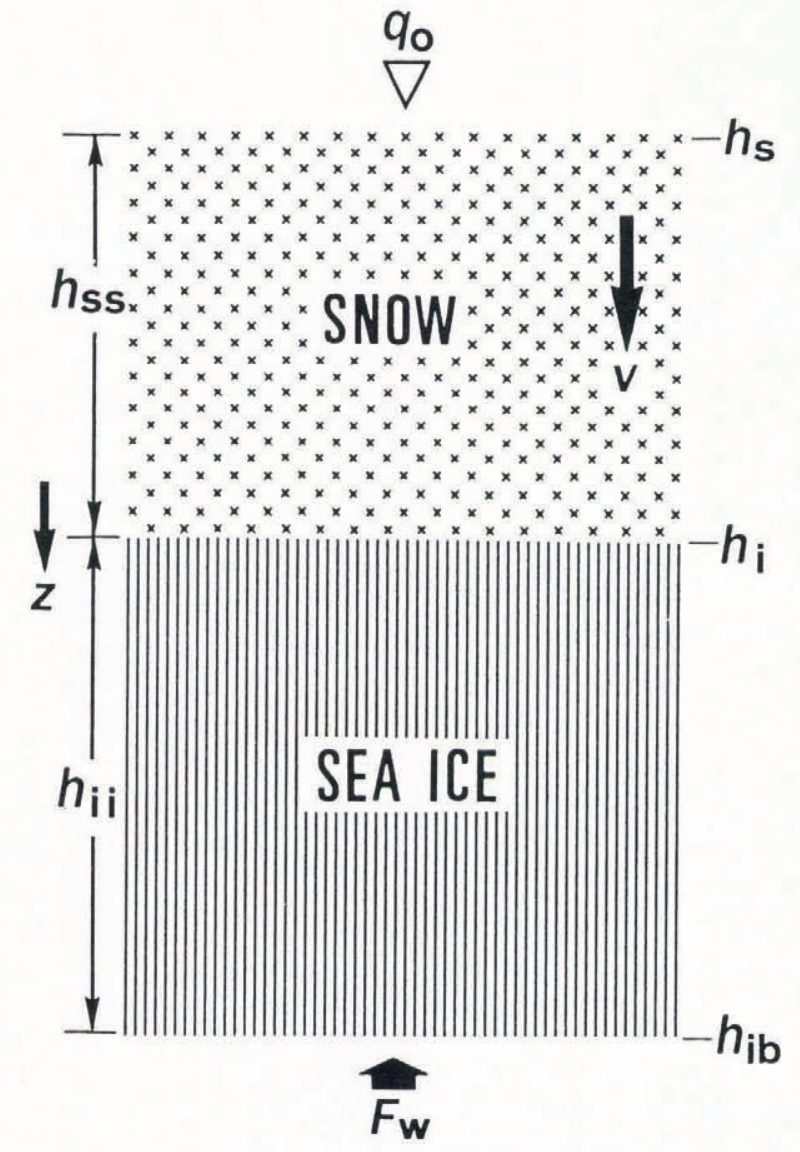

Fig. 1. Notation of snow-covered sea-ice model.

$$
F_{\mathrm{R}}=\frac{q_{0}(1-\alpha) b}{1-\alpha^{2} \exp (-2 b h)}\{\exp (-b z)-b \exp [b(z-2 h)]\}
$$

where $q_{0}$ is initial short-wave flux of solar radiation and $h$ is the thickness of the snow or ice layer.

\section{Snow layer}

The snow temperature $\left(T_{8}\right)$ as a function of depth $(z)$ and time $(t)$ is given by the differential equation of heat and mass transfer for a non-uniform medium:

$$
\rho_{\mathrm{s}} c_{\mathrm{s}}\left(\frac{\partial T_{\mathrm{s}}}{\partial t}+v \frac{\partial T_{\mathrm{s}}}{\partial z}\right)=\frac{\partial}{\partial z}\left(\lambda_{\mathrm{s}} \frac{\partial T_{\mathrm{s}}}{\partial z}\right)+F_{\mathrm{Rs}}+F_{\mathrm{e}}+F_{\mathrm{ms}},
$$

where $c_{8}, \rho_{8}$ and $\lambda_{8}$ are the specific heat, density and thermal conductivity of snow, respectively; $F_{\mathrm{R}}$ is the solar radiative heat source function given by Equation (1); $F_{\mathrm{e}}$ is the evaporation-condensation function; $F_{\mathrm{m}}$ is the melting-freezing function; and $v$ is rate of displacement of the snow medium. Subscript $\mathbf{s}$ is related to snow.

The process of evaporation-condensation in a snow medium is described by the equation for saturated vapor pressure (Krass and Merzlikin, 1990)

$$
P_{\text {sat }}(T)=P_{\text {os }} \exp [17(T-273) / T],
$$

where $P_{\text {sat }}$ and $P_{\mathrm{OB}}$ are the pressures of water vapor at temperatures $T$ and $0^{\circ} \mathrm{C}$ respectively. Consequently, the dimensionless saturated water vapor content in snow, $e_{\mathrm{v}}$ (per unit volume), is given by a similar equation:

$$
e_{\mathrm{v}}=e_{\mathrm{vo}} \exp [17(T-273) / T] \text {. }
$$

$P_{\text {sat }}$ as a function of temperature $(T)$ is

$$
\begin{aligned}
\log \left[P_{\text {sat }}(T)\right]= & \frac{-2445.5646}{T}+8.2312 \log T-0.01677 T \\
& +\left(1.20514 \times 10^{-5}\right) T^{2}-4.63227
\end{aligned}
$$

with $P_{\text {sat }}$ in $\mathrm{Pa}$ and $173 \mathrm{~K}<T<273 \mathrm{~K}$ (Mukai, 1986).

Under the supposition of thermodynamical equilibrium of evaporation-condensation processes, the function may be represented (Krass and Merzlikin, 1990)

$$
F_{\mathrm{e}}=L_{\mathrm{v}} \rho_{\mathrm{i}} \frac{\partial e_{\mathrm{v}}}{\partial t}=L_{\mathrm{v}} \rho_{\mathrm{i}} \frac{\partial e_{\mathrm{v}}}{\partial T} \frac{\partial T}{\partial t},
$$

where $L_{\mathrm{v}}$ is latent heat of evaporation and $\rho_{\mathrm{i}}$ is the density of ice.

The process of internal melting in snow (or opposite freezing of melt zones) is described by a function of phase transition

$$
F_{\mathrm{m}}=L_{\mathrm{m}} \rho_{\mathrm{i}} \frac{\partial e_{\mathrm{ms}}}{\partial t},
$$

where $L_{\mathrm{m}}$ is latent heat of phase transition ice-water and $e_{\mathrm{mg}}$ is dimensionless water content in snow (per unit of volume).

As is well known from numerous field observations, snow density varies strongly during the winter season. The basic physical properties of snow also vary, namely optical parameters, specific heat and thermal conductivity. The main reason for such density variations is the process of compaction of the snow medium due to gravity and surface wind load. Compaction of snow is described by (Mellor, 1975)

$$
\frac{\partial v_{\mathrm{c}}}{\partial z}=\frac{P}{\eta}
$$

where $v_{\mathrm{c}}$ is velocity of downward compaction, $P$ is normal pressure and $\eta$ is viscous analogy of compression modulus. From (7) we obtain for a snow compaction velocity

$$
v_{\mathrm{c}}=\int_{\mathrm{h}_{\mathrm{s}}}^{\mathrm{z}} \frac{P}{\eta} \mathrm{d} z, P=\int_{\mathrm{h}_{\mathrm{s}}}^{\mathrm{z}} \rho_{\mathrm{s}} g \mathrm{~d} z+P_{\mathrm{w}},
$$

where $P_{\mathrm{w}}$ is wind load pressure on the snow surface.

$\eta$ is strongly dependent on snow density (Mellor, 1975), and an approximation formula may be derived in the form

$$
\log \eta=\mathrm{A} \rho_{\mathrm{B}},
$$

where $A$ is determined by snow type.

The variations in snow density may be evaluated from the compaction formulae in the following way. Compaction displacement of the snow medium is given by integration of Equation (7) as

$$
u(t)=\int_{0}^{t} v_{c} \mathrm{~d} t .
$$

Snow-density increases and variations of snow-thermophysical parameters as influenced by compaction processes are calculated from

$$
\begin{gathered}
p=p_{\mathrm{o}}-\frac{u}{h+u}, \rho_{\mathrm{s}}=\rho_{\mathrm{i}}(1-p), p_{\mathrm{o}}=1-\frac{\rho_{\mathrm{so}}}{\rho_{\mathrm{i}}}, \\
c_{\mathrm{B}}=c_{\mathrm{i}}(1-p), \quad \lambda_{\mathrm{B}} \simeq \lambda_{\mathrm{i}}(1-p),
\end{gathered}
$$


where $p$ and $p_{0}$ are current porosity and initial porosity of snow, respectively, and $\rho_{\mathrm{so}}$ is initial snow density.

The velocity of snow displacement, which is included in Equation (2), is calculated from

$$
v(z, t)=v_{\mathrm{c}}\left(h_{\mathrm{b}}\right)-v_{\mathrm{c}}(z),
$$

where $h_{\mathrm{b}}$ is the lower surface of snow cover and is equal to $h_{\mathrm{i}}$ shown in Figure 1. The second term on the left side of Equation (2) containing $v$ is responsible for the heat-mass transfer in the snow medium. This may cause substantial changes in recent theoretical prognostications of sea ice temperature field.

\section{Sea ice}

The temperature of sea ice as a function of $z$ and $t$ and $v$ is given by a heat-transfer equation, which includes internal absorption, scattering of short-wave solar radiation, and transition of ice-brine phase equilibrium as follows

$$
c_{\mathrm{i}} \rho_{\mathrm{i}} \frac{\partial T_{\mathrm{i}}}{\partial t}=\frac{\partial}{\partial z}\left(\lambda_{\mathrm{i}} \frac{\partial T_{\mathrm{i}}}{\partial t}\right)+F_{\mathrm{Ri}}+F_{\mathrm{mi}},
$$

where subscript $\mathrm{i}$ refers to ice. The function for radiative source is written as (Krass and Merzliken, 1990)

$$
\begin{aligned}
& F_{\mathrm{Ri}}=F_{\mathrm{oi}}\left\{\exp \left(-b_{\mathrm{i}} z\right)-b_{\mathrm{i}} \exp \left[b_{\mathrm{i}}\left(z-h_{\mathrm{ii}}\right)\right]\right\} \\
& F_{\mathrm{oi}}=\frac{q_{\mathrm{o}}\left(1-\alpha_{\mathrm{s}}\right) b_{\mathrm{s}}}{1-\alpha_{\mathrm{s}}^{2} \exp \left(-2 b_{\mathrm{s}} h_{\mathrm{ss}}\right)} \frac{b_{\mathrm{i}}}{1-\alpha_{\mathrm{i}}^{2} \exp \left(-2 b_{\mathrm{i}} h_{\mathrm{ii}}\right)},
\end{aligned}
$$

where $h_{\mathrm{ss}}$ and $h_{\mathrm{ii}}$ are the thickness of snow cover and seaice layers respectively. Equation (14) takes into account attenuation of solar radiation flux in the overlying snow cover. If snow cover is absent, Equation (1) can be used with substitution of values $b_{\mathrm{i}}$ and $\alpha_{\mathrm{i}}$ for the sea ice.

The parameters for sea ice depend strongly on its salinity $S$ and temperature $T$. The formulae for specific heat and heat of fusion of sea ice, respectively, are (Ono, 1967)

$$
\begin{aligned}
c_{\mathrm{i}}= & 0.505+0.0018 T+4.3115 S / T^{2} \\
& -0.0008 S+0.00002 S T \quad\left(\mathrm{calg}^{-1}{ }^{\circ} \mathrm{C}^{-1}\right), \\
L_{\mathrm{mi}}= & 79.68-0.505 T-0.0273 S \\
& +4.3115 S / T+0.0008 S T \\
& -0.0009 T^{2} \quad\left(\mathrm{calg}^{-1}\right) .
\end{aligned}
$$

Thermal conductivity of sea ice is approximated by (Untersteiner, 1961)

$$
\lambda_{\mathrm{i}}=\lambda_{\mathrm{io}}+\frac{\beta S}{T_{\mathrm{i}}},
$$

where $\beta=0.13 \mathrm{~W} \mathrm{~m}^{-1}, S$ is ppt and $T_{\mathrm{i}}$ is ${ }^{\circ} \mathrm{C}$.

The temperature of phase transition also depends on salinity in accordance with the highly accurate equation (Fofonoff and Millard, 1983)

$$
T_{\mathrm{mi}}=a_{\mathrm{o}} S+a_{1} S^{\frac{3}{2}}+a_{2} S^{2}
$$

where

$$
\begin{gathered}
a_{0}=-0.0575, a_{1}=1.710523 \times 10^{-3}, \\
a_{2}=-2.154996 \times 10^{-4} .
\end{gathered}
$$

As the upward brine permeability of sea ice exceeds downward permeability, especially in the ice growing season (Ono and Kasai, 1985), the snow on the sea ice becomes salty due to the percolation of brine into the snow cover.

Brine content in sea ice and snow cover is of interest for research in sea-ice properties. Measurements have shown that this amount depends on the temperature and salinity of sea ice. Frankenstein and Garner (1967) developed empirical equations on the relative volume of brine in sea ice as a function of $S$ and $T$ as follows:

$$
\begin{aligned}
& v_{\mathrm{b}}=S(45.917 / T+0.930)-2.0>T>-8.2^{\circ} \mathrm{C}, \\
& v_{\mathrm{b}}=S(43.795 / T+1.189)-8.2>T>-22.9^{\circ} \mathrm{C} .
\end{aligned}
$$

If the temperature of sea ice is known from solving the thermal-regime problem and the salinity distribution in ice is known as input data, then the content of brine in sea ice may be calculated from (19).

\section{BOUNDARY AND INITIAL CONDITIONS}

To solve the thermal-regime problem in snow-covered sea ice, it is necessary to define boundary and initial conditions. Initial conditions correspond to the initial state of the natural system, which is supposed to be known. Boundary conditions correspond to the external influence of atmosphere and oceanic environments. Boundary conditions for the problem are separated into three groups: the snow-cover surface, intermediate surface between snow and sea ice, and the bottom surface of sea ice.

\section{Snow surface}

Heat and mass balance are described by the next equations. In the case of a negative snow-surface temperature, i.e. $T_{8}$ is below melting point, we obtain the equation

$$
-\lambda_{\mathrm{s}} \frac{\partial T_{\mathrm{s}}}{\partial z}=B\left(T_{\mathrm{a}}-T_{\mathrm{s}}\right)+\epsilon_{\mathrm{s}} C_{\mathrm{o}}\left(T_{\mathrm{a}}^{4}-T_{\mathrm{s}}^{4}\right)-\dot{Z}_{\mathrm{e}} L_{\mathrm{v}} \rho_{\mathrm{i}},
$$

where $B$ is coefficient of turbulent heat exchange between the atmosphere and the snow surface, $T_{\mathrm{a}}$ is the air temperature, $C_{\mathrm{o}}$ is the Stefan Boltzman constant $(5.67 \times$ $10^{-8} \mathrm{~W} \mathrm{~m}^{-2} \mathrm{~K}^{4}$ ), $\epsilon$ is the emissivity of snow (approximately equal to 1 ), and $\dot{Z}_{\mathrm{e}}$ is the evaporation-condensation rate on snow surface. Equation (20) shows that the surfacetemperature gradient is determined by turbulent heat exchange between the snow and air by long-wave radiation from the atmosphere and reradiation from snow surface, and by latent heat of snow evaporation (or condensation of water from the air) upon the snow surface. The amount of $\dot{Z}_{\mathrm{e}}$ may be evaluated by a semiempirical formula (Pavlov, 1984)

$$
\begin{aligned}
\dot{Z}_{\mathrm{e}}= & \left(3.6^{-1} \times 10^{-12}\right)\left(75+49 u_{2}\right) \\
& \left\{P_{\mathrm{o} 6} \exp [17(T-273) / T]-P_{\mathrm{a}}\right\},
\end{aligned}
$$

where $u_{2}$ is speed of wind at the height of $2 \mathrm{~m}$ over snow 
surface, $P_{O B}$ is pressure of saturated vapor at the temperature $0^{\circ} \mathrm{C}$ upon a flat, icy surface and $P_{\mathrm{a}}$ is pressure of water vapor in the air.

If the snow surface temperature reaches melting point, Equation (20) must be replaced with the condition of melting, $T_{\mathrm{s}}=0^{\circ} \mathrm{C}(273 \mathrm{~K})$,

$$
\begin{aligned}
L_{\mathrm{m}} \rho_{\mathrm{s}} \dot{Z}_{\mathrm{ms}} & =B\left(T_{\mathrm{a}}-T_{\mathrm{s}}\right)+\epsilon_{\mathrm{s}} C_{\mathrm{o}}\left(T_{\mathrm{a}}^{4}-T_{\mathrm{s}}^{4}\right)-\dot{Z}_{\mathrm{e}} L_{\mathrm{v}} \rho_{\mathrm{i}} \\
& +\lambda_{\mathrm{s}} \frac{\partial T_{\mathrm{s}}}{\partial z},
\end{aligned}
$$

where $\dot{Z}_{\mathrm{ms}}$ is rate of melting of snow surface.

\section{Surface of sea ice}

In the absence of snow cover, Equations (20) and (21a) are valid for the bare sea ice surface, with the replacement of subscript $\mathrm{s}$ by subscript $\mathrm{i}$ (formal substitution of sea ice parameters for snow parameters).

Two possible variants of conditions upon the boundary between snow cover and sea ice need to be defined. If the temperature on this surface is below the melting point (Equation (17)) heat fluxes from snow and sea ice are conserved:

$$
z=h_{\mathrm{b}}(t), \quad-\lambda_{\mathrm{s}} \frac{\partial T_{\mathrm{s}}}{\partial z}=-\lambda_{\mathrm{i}} \frac{\partial T_{\mathrm{i}}}{\partial z} .
$$

If the sea ice surface temperature reaches the melting point (Equation (17)) the boundary condition (22) can be replaced by the corresponding equation for the melting of sea ice

$$
T=T_{\mathrm{mi}}, \quad \lambda_{\mathrm{i}} \frac{\partial T_{\mathrm{i}}}{\partial z}-\lambda_{\mathrm{s}} \frac{\partial T_{\mathrm{s}}}{\partial z}=L_{\mathrm{mi}} \rho_{\mathrm{i}} \dot{Z}_{\mathrm{b}},
$$

where $L_{\mathrm{mi}}$ and $\lambda_{\mathrm{i}}$ are determined by Equations (15) and (16), respectively, and $\dot{Z}_{\mathrm{b}}$ is the melting rate of the sea ice surface. In the case where the sea ice surface temperature rises to $0^{\circ} \mathrm{C}$, Equation (22a) is replaced by the following conditions of snow and ice melting (brackets denote boundary jump of heat flux):

$$
\begin{aligned}
& T=0^{\circ} \mathrm{C},\left(-\lambda \frac{\partial T}{\partial z}\right)_{\mathrm{sb}}=L_{\mathrm{m}} \rho_{\mathrm{s}} \dot{Z}_{\mathrm{sb}} \\
& T=T_{\mathrm{mi}},\left(-\lambda \frac{\partial T}{\partial z}\right)_{\mathrm{ib}}=L_{\mathrm{mi}} \rho_{\mathrm{i}} \dot{Z}_{\mathrm{ib}}
\end{aligned}
$$

where $\dot{Z}_{\mathrm{sb}}$ is the melting rate on the bottom of snow and subscript $b$ refers to the brine layer on the sea-ice surface.

The melting of sea ice from above produces lowsalinity brine which may occur on the sea-ice surface for a finite time and causes a drop of sea-ice salinity due to brine wash out. Meltwater from the snow layer covering the sea ice leads to brine dilution, thus raising its freezing temperature. A relatively small temperature drop may cause formation on the top of the sea-ice cover. This process is an essential feature of sea-ice cover evolution, which may be described by the following equations. The equation of brine-layer salinity evolution is used in the form

$$
\dot{S}_{\mathrm{b}}+S_{\mathrm{b}}\left(\dot{Z}_{\mathrm{i}}+\dot{Z}_{\mathrm{sb}}\right) \frac{1}{\ell_{\mathrm{b}}}=\frac{S_{\mathrm{i}} \dot{Z}_{\mathrm{i}}}{\ell_{\mathrm{b}}}, \quad \ell_{\mathrm{b}}=\int_{0}^{t}\left(\dot{Z}_{\mathrm{sb}}+\dot{Z}_{\mathrm{i}}\right) \mathrm{dt},
$$

where $\ell_{\mathrm{b}}$ is the thickness of the brine layer on the sea ice surface. Notice here that if $\dot{Z}_{\mathrm{i}}, \dot{Z}_{\mathrm{sb}}$ and $\ell_{\mathrm{b}}$ variations are relatively slow, then the approximate solution of Equation (23) may be derived in an analytical form as

$$
\begin{aligned}
& S_{\mathrm{b}}=\exp {\left[-\int \frac{1}{\ell_{\mathrm{b}}}\left(\dot{Z}_{\mathrm{i}}+\dot{Z}_{\mathrm{sb}}\right) \mathrm{d} t\right] } \\
&\left\{\int \frac{1}{\ell_{\mathrm{b}}} S_{\mathrm{i}} \dot{Z}_{\mathrm{i}} \exp \left[\int \frac{1}{\ell_{\mathrm{b}}}\left(\dot{Z}_{\mathrm{i}}+\dot{Z}_{\mathrm{sb}}\right) \mathrm{d} t\right] \mathrm{d} t+\mathrm{C}\right\},
\end{aligned}
$$

where the constant $\mathrm{C}$ is determined from the initial conditions.

\section{Bottom of sea ice}

The growth or decrease of the sea-ice thickness from below is determined completely by the heat-mass balance on the bottom of ice. The variation rate of the sea-ice bottom is due to the melting of sea ice or freezing of sea water as given by

$$
T=T_{\mathrm{mi}}, \quad \lambda_{\mathrm{i}} \frac{\partial T_{\mathrm{i}}}{\partial z}-F_{\mathrm{w}}=\rho_{\mathrm{i}} L_{\mathrm{mi}}(S) \dot{h_{\mathrm{ib}}},
$$

where $F_{\mathrm{w}}$ is the heat flux from the sea water to ice and $\dot{h}_{\mathrm{ib}}$ is the rate of melting or freezing.

\section{Movement of snow surface}

The total displacement of the snow surface is due to six effects: (1) surface evaporation-condensation $\left(\dot{Z}_{\mathrm{e}}\right)$; (2) surface melting of snow $\left(\dot{Z}_{\mathrm{ms}}\right)$; (3) snowfall $\left(a_{\mathrm{h}}\right)$; (4) melting within snow cover $\left(\dot{e}_{\mathrm{ms}}\right) ;(5)$ snow melting on the boundary between snow and sea ice; and (6) compaction of snow. Therefore, the rate of movement of the snowcover upper boundary $\dot{\mathrm{h}}_{\mathrm{B}}$ is determined by

$$
\dot{h}_{\mathrm{s}}=\dot{Z}_{\mathrm{e}}+\dot{Z}_{\mathrm{ms}}+\dot{Z}_{\mathrm{s}}+\epsilon \int_{\mathrm{h}_{\mathrm{s}}}^{\mathrm{h}_{\mathrm{b}}}\left(\frac{\rho_{\mathrm{i}}}{\rho_{\mathrm{s}}}\right) \dot{e}_{\mathrm{ms}} \mathrm{d} z+v_{\mathrm{c}}\left(h_{\mathrm{b}}\right)+a_{\mathrm{h}} \text {, }
$$

where $\epsilon$ is the defect of volume under ice-water phase transition. Here, it is necessary to input the snowfall density as a function of time

$$
\rho_{\mathrm{ah}}=\rho_{\mathrm{fs}}(t) .
$$

\section{Initial conditions}

The thermal regime for the "snow-covered sea-ice" system is a time-dependent problem. Therefore, it is necessary to define the initial state of the system. The initial time corresponding to $t=0$ may be chosen arbitrarily; this choice depends on the evolutionary stage of the system which is to be used as the initial state. The set of initial conditions contains the following equations:

$$
\begin{aligned}
t=0 ; T_{\mathrm{s}} & =T_{\mathrm{so}}(z), \quad h_{\mathrm{so}} \leq z<h_{\mathrm{bo}} ; T_{\mathrm{i}}=T_{\mathrm{io}}(z), \\
h_{\mathrm{bo}} & \leq z<h_{\mathrm{io}} ; h_{\mathrm{s}}=h_{\mathrm{so}}=0, h_{\mathrm{ib}}=h_{\mathrm{bo}}, \\
h_{\mathrm{i}} & =h_{\mathrm{io}} ; \quad S_{\mathrm{b}}=S_{\mathrm{bo}}, \quad \ell_{\mathrm{b}}=\ell_{\mathrm{bo}}, \\
F_{\mathrm{ms}} & =F_{\mathrm{mso}}(z), h_{\mathrm{so}} \leq z<h_{\mathrm{bo}} ; \quad F_{\mathrm{mi}}=F_{\mathrm{mio}}(z), \\
h_{\mathrm{bo}} & \leq z<h_{\mathrm{io}} .
\end{aligned}
$$




$$
\begin{array}{cc}
\rho & k \times 10^{6} \\
\mathrm{~kg} \mathrm{~m}^{-3} & \mathrm{~m}^{2} \mathrm{~s}^{-1}
\end{array}
$$

$8 \mathrm{~h}$

Periods of air temperature oscillations

\begin{tabular}{rrrrrrrrr}
1. & 50 & 0.085 & 102.9 & 36.4 & 29.7 & 21.0 & 12.1 & 6.6 \\
2. & 100 & 0.165 & 72.7 & 25.7 & 21.0 & 14.8 & 8.6 & 4.7 \\
3. & 200 & 0.330 & 51.4 & 18.2 & 14.8 & 10.5 & 6.1 & 3.3 \\
4. & 300 & 0.495 & 42.0 & 14.8 & 12.1 & 8.6 & 4.9 & 2.7 \\
5. & 400 & 0.660 & 36.4 & 12.9 & 10.5 & 7.2 & 4.3 & 2.3 \\
\hline
\end{tabular}

\section{PECULIARITIES OF THE PROBLEM}

The problem consisting of Equations and conditions (1) (27) represents a mathematical model of the thermal regime of "snow-covered sea-ice" system, which takes into account the following processes in snow and sea ice: compaction of snow cover under gravity and wind load; evaporation-condensation in snow and melting within a snow and sea-ice media; dependence of sea-ice melting upon salinity; influence of optical characteristics of snow and sea ice upon the thermal regime of the system; and a non-stationary snow-cover thickness due to snowfall and compaction.

The developed problem is comprehensive. It allows us to develop simulations and to study theoretically the thermal regime with a wide range of its properties and peculiarities - from origination of young sea ice to its internal melting caused by warming within the snow cover. The model appears useful as a tool to study sea-ice cover behavior, especially the rapid disintegration during the summer season.

One of the most important features of the problem is consideration of the snow-compaction process. Thermophysical parameters of snow are changeable under an increase in density: snow perviousness to air temperature oscillations increases with its densification. Attenuation of the amplitude $A_{\mathrm{a}}$ of the air temperature oscillation during the period $t_{\mathrm{o}}$ in snow at depth $z$ is given by

$$
A_{\mathrm{z}}=A_{\mathrm{a}} \exp \left[-z \sqrt{\left(\pi / k t_{0}\right)}\right],
$$

where $k$ is thermal diffusivity of snow. The decrement of the attenuation is

$$
D=\sqrt{\left(\pi / k t_{0}\right)} .
$$

Table 1 shows the dependence the decrement in snow density has on various periods of air temperature oscillations. Densities of $50-100 \mathrm{~kg} \mathrm{~m}^{-3}$ correspond to fresh-fallen snow; densities in excess of $300 \mathrm{~kg} \mathrm{~m}^{-3}$ indicate densified snow after compaction. The higher the snow density, the less decrement in attenuation of airtemperature oscillations within snow medium.

In accordance with Table 1 , Table 2 demonstrates the penetration of air temperature oscillations to the bottom of the snow cover $(10 \mathrm{~cm}$ thick) resulting from calculations with Equation (29).

As is evident from Table 2, fresh-fallen snow is almost impervious to the air-temperature oscillations within periods of $12 \mathrm{~h}$ and less. Along with densification, permeability of snow increases considerably: so, within a $10 \mathrm{~cm}$ thick snow cover of high density, the amplitude of air-temperature oscillations for $8-12 \mathrm{~h}$ and $24 \mathrm{~h}$ are reduced to about $30 \%$ and $50 \%$, respectively.

The model includes a set of measured parameters which are to be input. They may be separated into two

\begin{tabular}{|c|c|c|c|c|c|c|c|c|}
\hline \multicolumn{9}{|c|}{$\rho$} \\
\hline & $\mathrm{kg} \mathrm{m} \mathrm{m}^{-3}$ & $1 \mathrm{~h}$ & $8 \mathrm{~h}$ & $12 \mathrm{~h}$ & $1 \mathrm{~d}$ & $3 d$ & $10 \mathrm{~d}$ & $1 \mathrm{month}$ \\
\hline 1. & 50 & 0.003 & 3 & 5 & 12 & 30 & 52 & 68 \\
\hline 2. & 100 & 0.1 & 8 & 12 & 23 & 42 & 63 & 76 \\
\hline 3. & 200 & 0.6 & 16 & 23 & 35 & 54 & 72 & 83 \\
\hline 4. & 300 & 1.5 & 23 & 30 & 42 & 61 & 76 & 85 \\
\hline 5. & 400 & 2.6 & 28 & 35 & 49 & 65 & 80 & 87 \\
\hline
\end{tabular}
groups. The first, corresponding to the "snow-cover and

Table 2. Penetration of air temperature oscillations to the bottom of $10 \mathrm{~cm}$-thick layer of snow (per cent of the amplitude of the air temperature) 
sea-ice" system, contains the following amounts: thermophysical parameters of snow and sea ice; mechanical parameter of snow (compressional viscosity); optical parameters of snow and ice; and thermodynamical parameters of sea ice. The second group includes only parameters of the external conditions, namely, effect of the climate and sea water upon sea ice: air temperature; solar radiation flux; and heat exchange between snowcovered sea-ice system and environment.

The theoretical study of the thermal regime of snowcovered sea ice consists mainly in testing system responses to various sets of second-group parameters. The most interesting is the study of select responses of the sea-ice cover to short-term and long-term climatic variations.

\section{A CASE STUDY OF "WARM SEA ICE"}

The general problem of the thermal regime in snowcovered sea ice as formulated above may be simplified in the case of seasonal sea ice covering moderate seas, such as the sea of Okhotsk. Since deviations in the temperature of sea ice from the melting point are negligible, one may keep the first order of magnitude terms in the equation of thermal state (13) by the elimination of terms including temperature. Equation (13) is then reduced to the form

$$
F_{\mathrm{Ri}}+F_{\mathrm{mi}}=0
$$

i.e. the internal thermal regime of sea ice is determined by the equilibrium between absorption of solar-radiation flux and melting of sea ice. The heat and mass balance on the surface and on the bottom of sea-ice cover is defined by the boundary conditions of Equations (20)-(24) as before, but Equation (22) has to be omitted, and a simplified form of Equation (22a) describes the thermal regime of the sea-ice surface as follows

$$
-F_{\mathrm{w}}+\lambda_{\mathrm{s}} \frac{\partial T_{\mathrm{s}}}{\partial z}=L_{\mathrm{mi}} \rho_{\mathrm{i}} \dot{Z}_{\mathrm{b}}
$$

In the case of a bare sea ice surface, the boundary condition of heat exchange between sea ice and air from Equation (20) describes the rate of surface melting,

$$
\begin{aligned}
& L_{\mathrm{mi}} \rho_{\mathrm{i}} \dot{Z}_{\mathrm{mi}}=B\left(T_{\mathrm{a}}-T_{\mathrm{i}}\right) \\
& \quad+\epsilon_{\mathrm{i}} C_{\mathrm{o}}\left(T_{\mathrm{a}}^{4}-T_{\mathrm{i}}^{4}\right)-\dot{Z}_{\mathrm{e}} L_{\mathrm{v}} \rho_{\mathrm{i}}, T_{\mathrm{i}}=T_{\mathrm{m}} .
\end{aligned}
$$

The thermodynamical relations of dependencies of melting point and latent heat of sea ice upon salinity (Equations (15) and (17)) are valid as before.

\section{CONCLUSION}

We have attempted in this paper to develop a model of the thermal regime in snow-covered sea ice which is comprehensive and applicable to discovering a wide set of sea-ice behavioral peculiarities. The thermal regime, including internal phase transitions, is responsible for important mechanical parameters of sea ice, such as durability, fracture toughness and stability. The shortterm variations of air temperature and solar-radiation flux produce a thermal regime in sea ice which may contribute to drastic disintegrations of sea-ice cover.

The long-term climatic variations are apparently responsible for the stability of the sea ice cover on global scales. Output data from the model presented are available for use as input data for corresponding models of mechanical disturbance of the sea-ice cover.

\section{REFERENCES}

Fofonoff, N. P. and R. C. Millard, Jr. 1983. Algorithms for computation of fundamental properties of seawater. UNESCO Tech. Pap. Mar. Sci. 44.

Frankenstein, G. and R. Garner. 1967. Equations for determining the brine volume of sea ice from $-0.5^{\circ}$ to $-22.9^{\circ}$ C. 7. Glaciol., 6(48), 943-944.

Krass, M.S. and V.G. Merzlikin. 1990. Radiyatsionnaya teplofizika snega $i$ l'da [The radiative thermophysics of snow and ice]. Leningrad, Gidrometeoizdat.

Maykut, G. A. 1986. The surface heat and mass balance. In Untersteiner, N., ed. The geophysics of sea ice. New York, Plenum Press, 395-463.

Mellor, M. 1975. A review of basic snow mechanics. International Association of Hydrological Sciences Publication 114 (Symposium at Grindelwald 1974 - Snow Mechanics), 251-291.

Mukai, T. 1986. Analysis of a dirty water-ice model for cometary dust. Astron. Astrophys., 164, 397-407.

Ono, N. 1967. Specific heat and heat of fusion of sea ice. In Ōura, H., ed. Physics of snow and ice. Sapporo, Hokkaido University. Institute of Low Temperature Science, 599-610.

Ono, N. and T. Kasai. 1985. Surface layer salinity of young sea ice. Ann. Glaciol., 6, 298-299.

Pavlov, A. V. 1984. Energoobmen $v$ landshaftnoy sfere Zemli [Energy exchange in landscape sphere of the Earth]. Moscow, Nauka.

Untersteiner, N. 1961. On the mass and heat budget of Arctic sea ice. Arch. Meteorol. Geophys. Bioklimatol. et al., Ser. A, 12(2), 151-182.

The accuracy of the references in the text and in this list is the responsibility of the authors, to whom queries should be addressed. 